UCRL-JC-132974

PREPRINT

\title{
Compact, Low-Crosstalk, WDM Filter Elements for Multimode Ribbon Fiber Data Links
}

\author{
R.R. Patel \\ H.E. Garrett \\ M.A. Emanuel \\ M.C. Larson \\ M.D. Pocha \\ D.M. Krol \\ R.J. Deri \\ M.E. Lowry
}

This paper was prepared for submittal to the

Electronic Components and Technology Conference

San Diego, CA

June 1-4, 1999

\section{January 1999}

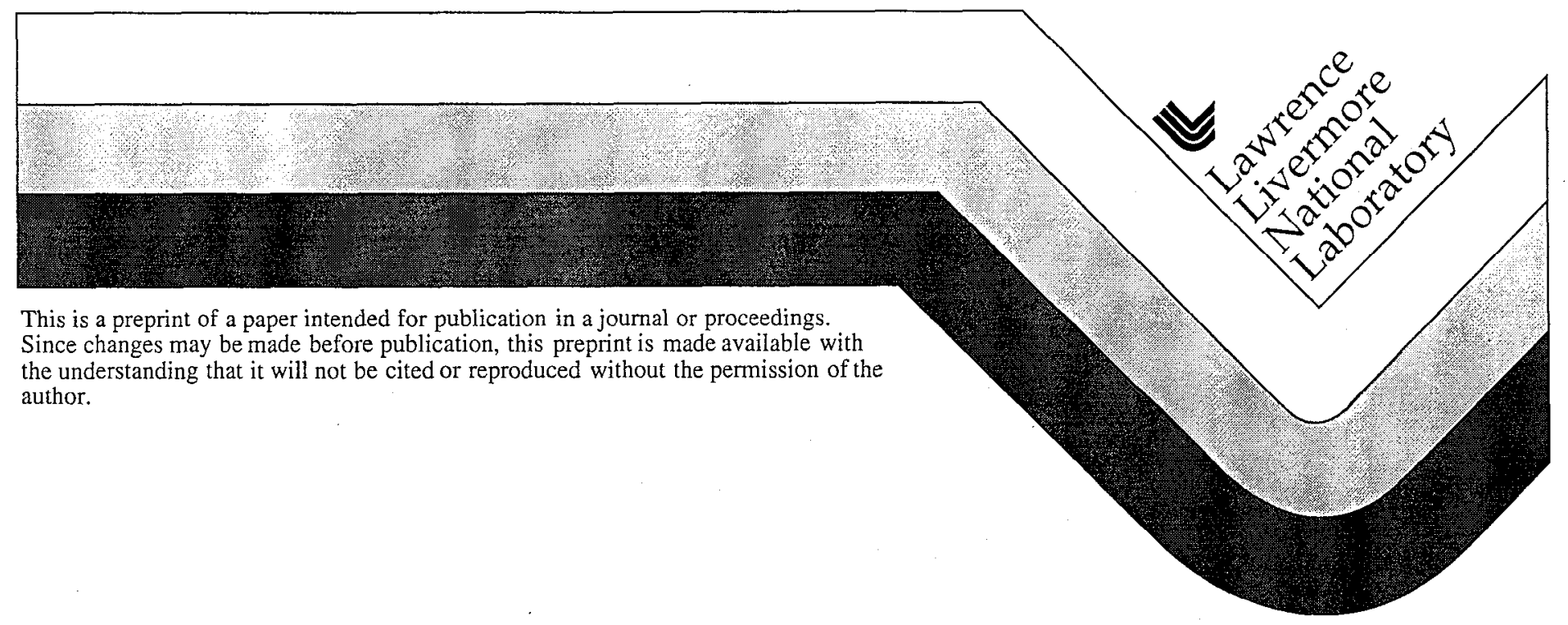




\section{DISCLAIMER}

This document was prepared as an account of work sponsored by an agency of the United States Government. Neither the United States Government nor the University of California nor any of their employees, makes any warranty, express or implied, or assumes any legal liability or responsibility for the accuracy, completeness, or usefulness of any information, apparatus, product, or process

disclosed, or represents that its use would not infringe privately owned rights. Reference herein to any specific commercial product, process, or service by trade name, trademark, manufacturer, or otherwise, does not necessarily constitute or imply its endorsement, recommendation, or favoring by the United States Government or the University of California. The views and opinions of authors expressed herein do not necessarily state or reflect those of the United States Government or the University of California, and shall not be used for advertising or product endorsement purposes. 


\title{
Compact, Low-Crosstalk, WDM Filter Elements for Multimode Ribbon Fiber Data Links
}

\author{
R. R. Patel, H. E. Garrett, M. A. Emanuel, M. C. Larson, \\ M. D. Pocha, D. M. Krol, R. J. Deri, and M. E. Lowry \\ Lawrence Livermore National Laboratory \\ 7000 East Ave., Livermore, CA, 94550 \\ (925) 422-9868 email: patel6@llnl.gov
}

\begin{abstract}
We have been developing the optical components for a source-routed wavelength division multiplexed (WDM) computer interconnect fabric that uses multi-mode fiber ribbon cable as the transmission medium. We are developing wavelength selectable VCSEL transmitters, interference filters, and a compact broadcast element.

Here we report on key results from our interference filter development activities. Our WDM filter approach is based upon post-market machining of the commercially available molded plastic "MT" fiber ribbon connector. We use III-IV semiconductors grown by MBE or MOCVD as the filter materials. The high indices of our thin film materials enable us to use multimode fiber and maintain narrow passbands without the need for micro-optics. We have fabricated both 2-port and 3-port devices based upon this approach. Our current work focuses on 2-port WDM filters suitable for a broadcast and select architecture. Our single-cavity FabryPerot (FP) filters have demonstrated insertion losses of $<2$ $\mathrm{dB}$ for $4 \mathrm{~nm}$ passbands. The maximum crosstalk suppression for the single-cavity FP filtcrs is $18 \mathrm{~dB}$

To improve crosstalk suppression beyond that attainable with the Lorentzian lineshapes of the single-cavity FP we have investigated some multiple-cavity Fabry-Perot (MC-FP) designs which have a spectral response with a flatter top and sharper passband edges. Filter passband edge sharpness can be quantified by the ratio of the filter $3 \mathrm{~dB}$ bandwidth to 18 $\mathrm{dB}$ bandwidth. This ratio is 0.48 for our multi-cavity filter, threc timcs sharper than the singlc-cavity FP devices. This device provides a $5 \mathrm{~nm}$ tolerance window for component wavelength variations (at $1 \mathrm{~dB}$ excess loss) and is suitable for $10 \mathrm{~nm}$ channel spacing with $23 \mathrm{~dB}$ crosstalk suppression between adjacent channels. The average insertion loss for the MC-FP devices is $1.6 \mathrm{~dB}$. (Average of insertion losses for the 12 fibers in a filter module.) Our current MC-FP filters have a $3-\mathrm{dB}$ width of $7.6 \mathrm{~nm}$. Fiber to fiber center wavelength variations within a typical filter module are less than $1 \mathrm{~nm}$. The MC-FP devices exhibit cross-talk suppression $>30 \mathrm{~dB}$ over a $100 \mathrm{~nm}$ span.
\end{abstract}

\section{Introduction}

Parallel optical interconnects based on multimode fiber (MMF) ribbon cable are emerging as a robust, highperformance data link technology which increases channel bandwidth by using linear MMF arrays [1]. While this technology has primarily been implemented as single wavelength point-to-point links, it can be significantly enhanced by wavelength division multiplexing (WDM). WDM enables both increased point-to-point bandwidth as well as more complex interconnect topologies and routing approaches that are particularly attractive for high performance computing platforms [2]. Exploiting the potential richness of WDM interconnects, however, also requires a low-loss routing fabric that includes small footprint wavelength selective filters. Low insertion loss is critical for this technology because the transceivers exhibit link power budgets well below that of telecom WDM systems and because the MMF cabling precludes the use of optical amplifiers. While high performance filters can be realized for single-fiber applications [3,4], achieving highperformance, small footprint devices with ribbon cable is significantly complicated by MMF's high numerical aperture $(\mathrm{NA}=0.275)$ and large core diameter $(62.5 \mu \mathrm{m})$.

We have developed both 2-port and 3-port filter modules assembled in simple, robust packages. Our 3-port modules are suitable for add/drop multiplexing while the 2-port devices are suitable for broadcast and select architectures. In this paper we present a summary of our 3-port filter module work [5], and we focus on our recent work with 2-port filter modules.

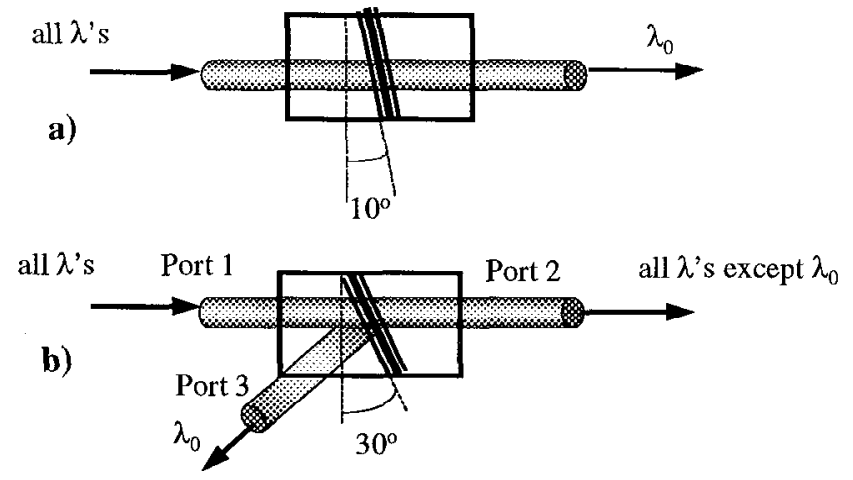

Fig. 1. Schematic of a) 2-port and b) 3-port filter modules

\section{Filter Packaging Issues}

Figure 1 shows the geometry of our filter modules while figure 2 provides a perspective view of a 2-port module to aid in visualization. A high refractive index $(>3.0)$ interference filter is sandwiched between parallel arrays of $12 \mathrm{MMFs}$ 


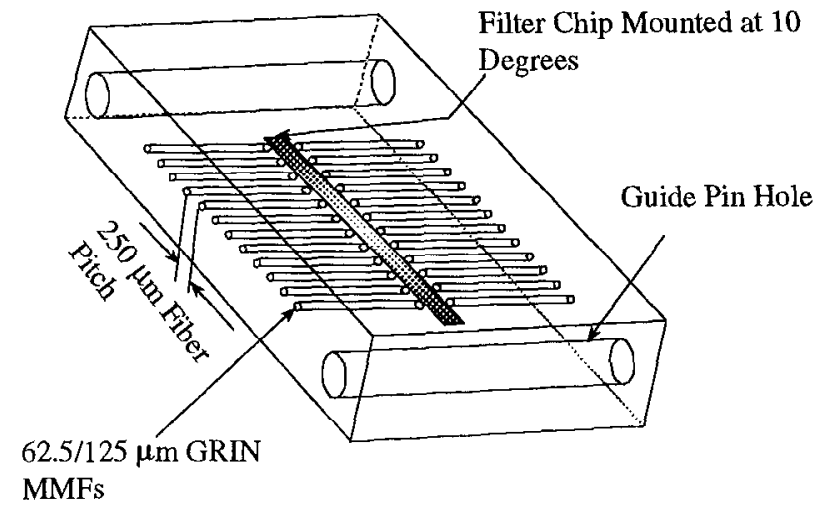

Fig. 2. Perspective view of a 2-port filter module packaged to be compatible with MT ferrules.

using an assembly of modified commercial 'MT' fiber ribbon connector ferrules[6]. The high filter index enables narrow passbands and sharp filter edges for the high MMF numerical aperture, without additional micro-optic collimation. Furthermore, for the 3-port modules, the use of high-index materials facilitates a large angle of incidence to ease optomechanical packaging while minimizing bandpass spreading and polarization sensitivity. The use of commercial ferrules minimizes device size $\left(2.7 \times 6.4 \times 7.6 \mathrm{~mm}^{3}\right.$ for 2-port modules and 5.7 X $6.4 \times 7.8 \mathrm{~mm}^{3}$ for 3-port modules), and simplifies assembly and packaging via passive alignment using guide pins. For the 2-port modules, alignment is $100 \%$ passive with the guide pins, while for the 3-port modules, the longitudinal alignment of port 3 along the port 1-2 axis is done by active alignment - this could be eliminated in production by a modified ferrule design. In addition, these modules mate directly with MT-terminated fiber ribbon cables without the need for additional connectors. The minimal complexity of the assembly, coupled with the plastic molding used to realize the ferrules, indicates the potential for cost-effective manufacture of such devices.

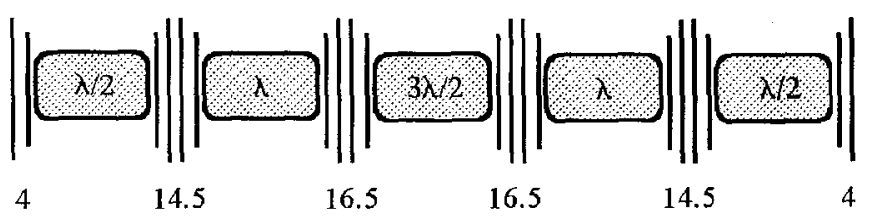

Fig. 3. Layer structure of the five cavity MC-FP filter. Shaded regions represent cavities of the indicated length (in terms of center wavelength $\lambda$ ). The number of bragg pairs in each reflector are also indicated. Fractional Bragg paits indicate extra low-index spacer layers.

\section{Filter Design}

Our 3-port devices employ a simple Bragg reflector consisting of 31 Bragg pairs as the wavelength selective element. In the 2-port configuration we have demonstrated both single cavity Fabry-Perot type filters and more complex multiple-cavity Fabry-Perot (MC-FP) filters. The filters use

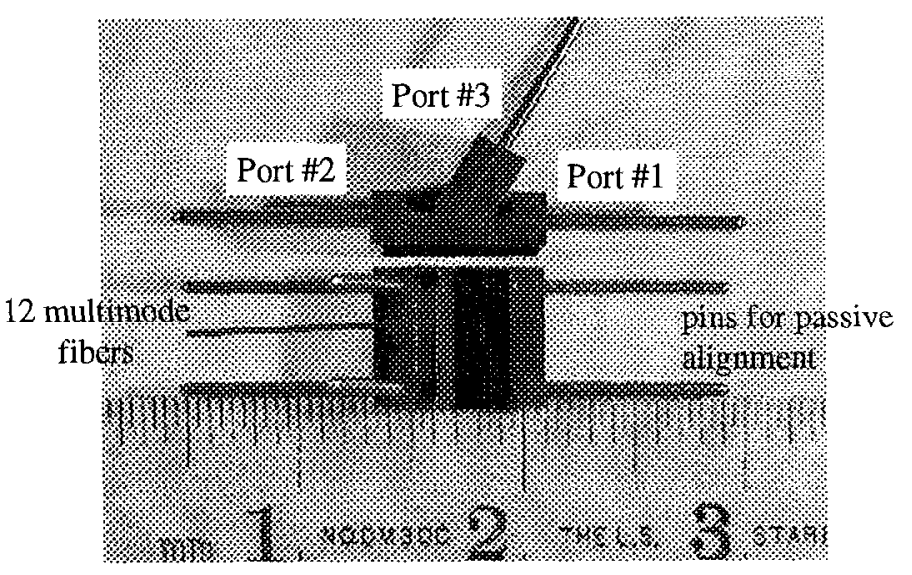

Fig. 4. Photograph of a 3-port filter module.

epitaxially grown $\mathrm{Al}_{0.15} \mathrm{Ga}_{0.85} \mathrm{As}$ and $\mathrm{AlAs}$ layer materials and were designed using standard methods [7]and/or by considering the filter as a one-dimensional photonic bandgap structure [8]. Our single cavity design has two Bragg mirrors, each consisting of 10 quarter-wave $\mathrm{Al}_{0.15} \mathrm{Ga}_{0.85} \mathrm{As} /$ AlAs Bragg pairs, spaced by a half-wave cavity layer. Our MC-FP filter design can be visualized as in figure 3, where numbers denote a Bragg reflector containing that number of Bragg pairs, and shaded regions show the thickness (in units of the optical center wavelength $\lambda$ ) of higher index $\mathrm{Al}_{0.15} \mathrm{Ga}_{0.85}$ As cavity layers inserted between the Bragg reflectors. Fractional Bragg pair values indicate lower index AlAs spacer layers inserted between the Fabry-Perot cavities in MC-FP designs. The filter layers are grown by metalorganic chemical vapor deposition on a GaAs substrate at $710{ }^{\circ} \mathrm{C}$ and 80 mbar. In addition to the actual filter layer structure described above, we also inserted $10 \mathrm{~nm}$ thick GaAs cap layers on both sides of the filter to minimize oxidation, and a $400 \mathrm{~nm} \mathrm{InGaP} \mathrm{etch-stop} \mathrm{layer} \mathrm{between} \mathrm{the} \mathrm{GaAs} \mathrm{cap}$ and the substrate to facilitate substrate removal.

\section{Module Assembly}

Filter modules are assembled from MT ferrule tips containing $62.5 \mu \mathrm{m}$ diameter core, graded index optical fibers. For the 2-port devices, ferrule endfacets are angled and polished at $10^{\circ}$ to improve return loss outside the filter passband. Filter material is then attached to one ferrule with transparent epoxy. To minimize diffractive and absorptive loss in these devices, the GaAs substrate material is removed using a selective wet etch consisting of $\mathrm{H}_{2} \mathrm{SO}_{4}: \mathrm{H}_{2} \mathrm{O}_{2}: \mathrm{H}_{2} \mathrm{O}$, followed by selective removal of the InGaP etch-stop layer using $\mathrm{H}_{3} \mathrm{PO}_{4}: \mathrm{HCl}$. Following these etches, the final assembly step is epoxy attachment of a second MT ferrule tip to the first, using guide pins to passively align all 12 fibers in the module simultaneously. For the 3-port modules, two ferrules are prepared at $30^{\circ}$ and the third at $60^{\circ}$. Furthermore, in the three port modules, once ports 1 and 2 are assembled the filter to fiber interface is exposed through a wafer saw cut. This allows introduction of the third port. During assembly, care is required in controlling the depth of the wafer saw cut and in the longitudinal positioning of the third port. Cut depth is monitored through microscope 
inspection while longitudinal alignment of the third port is currently done by active alignment. A photograph of a fully assembled 3-port filter module is given in figure 4 .

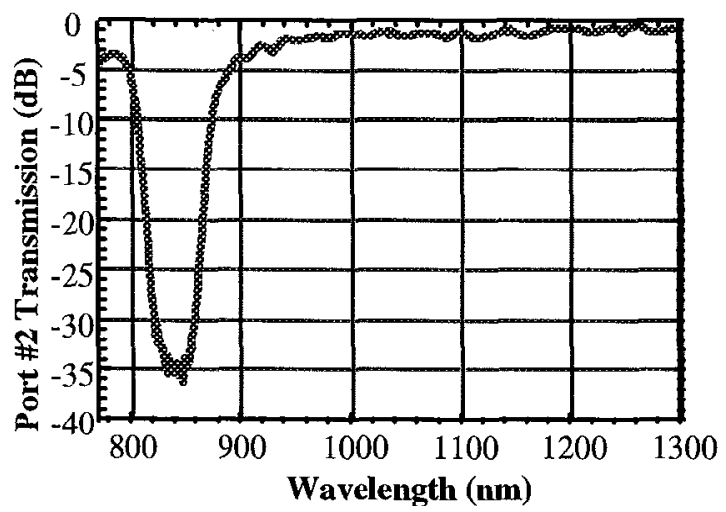

Fig. 5. Transmission characteristic for port \#2 of a typical 3-port filter module

\section{Filter Module Performance}

The resulting modules exhibit fiber-to-fiber separations of approximately $40 \mu \mathrm{m}$. Figure 5 shows the transmission characteristics for the straight-through light path (port \#2) of a typical 3-port device and figure 6 shows the transmission characteristics of our 2-port filter modules. These devices exhibit low fiber-to-fiber insertion loss. For the 3-port devices the insertion losses are $1.1 \mathrm{~dB}$ for the port 1-2 path and $1.3 \mathrm{~dB}$ for the port 1-3 path. It is critical that the port 1 2 insertion loss of 3 -port devices be minimized since this will ultimately limit the number of filters which can be serially cascaded in ring networks. For the 2-port devices insertion losses are 1.6 and $2.6 \mathrm{~dB}$ for $\mathrm{MC}$ - and single cavity devices, respectively. The higher insertion loss for the $4 \mathrm{~nm}$ bandpass filter is likely due to problems which occurred with the batch of optical epoxy being used at the time.

The 3-port filter devices were designed to have a $\sim 40 \mathrm{~nm}$ passband centered around $840 \mathrm{~nm}$ to be compatible with commercially available byte-wide transceivers, which are manufactured to moderate wavelength tolerances. The 2-port devices exhibit narrow passbands 7.6 full-width at half

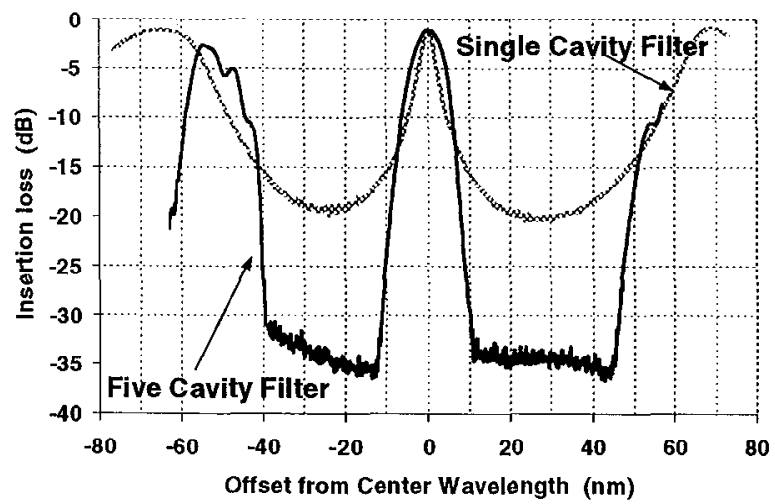

Fig. 6. Transmission spectra of 2-port filter modules plotted against offset from center wavelength to highlight passband shape differences. The actual center wavelengths are $823 \mathrm{~nm}$ and $848 \mathrm{~nm}$ for the multi- and single-cavity devices respectively

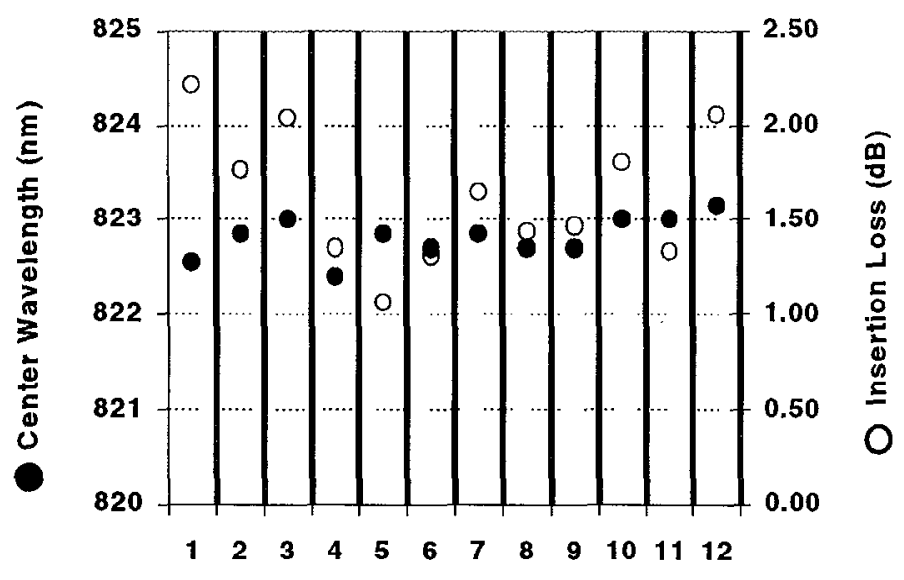

a)

Fiber Number

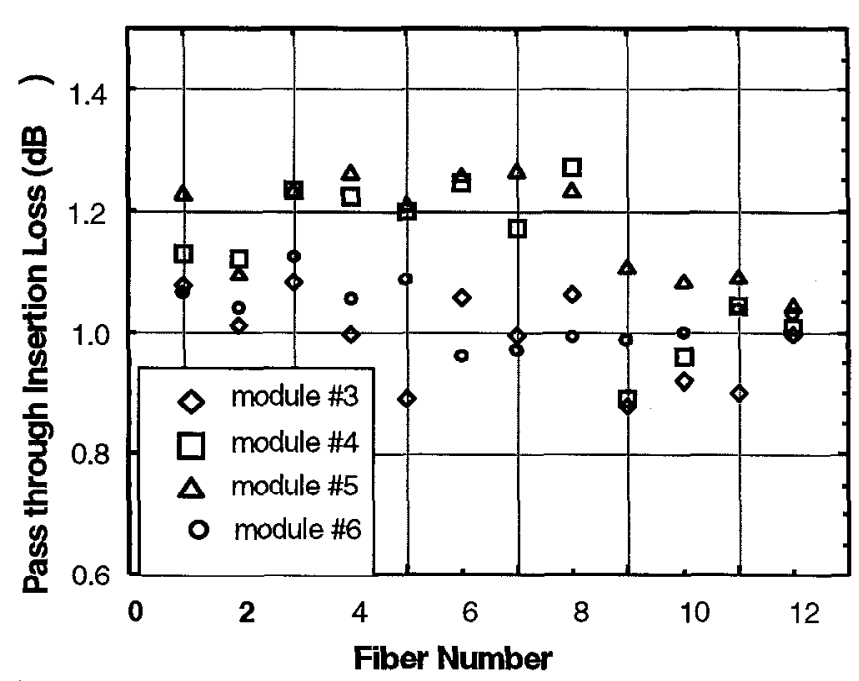

b)

Fig. 7. Uniformity data for filter modules. a) a 2-port multi-cavity filter, Center wavelength (solid markers) and insertion loss (open markers) for each fiber in the module. b) Insertion Loss for the straight-through path of several 3-port filter modules.

maximum (FWHM) for MC-FP, and $3.8 \mathrm{~nm}$ FWHM for single-cavity devices, respectively. Maintaining optimum filter FWHM with sharp pass-band edges is a key to achieving tightly spaced channels while allowing for transmitter drift and thermal variations. Fig. 6 shows that our multi-cavity design significantly improves these features. This improvement can be quantified by the ratio of filter -3 $\mathrm{dB}$ bandwidth to $-18 \mathrm{~dB}$ bandwidth, which improved from 0.13 to 0.46 for the multi-cavity design. The figure also shows improved crosstalk suppression for the multi-cavity design compared to the single cavity filter. The $35 \mathrm{~dB}$ suppression shown by fig. 6 is actually a lower limit set by the noise floor of our measurement system, rather than the filter characteristics. Based on this data, the MC-FP filters should provide crosstalk suppression $>23 \mathrm{~dB}$ for $10 \mathrm{~nm}$ channel spacing. 
For parallel optical interconnect applications, uniform filter performance must be maintained across all the fibers within a filter module. Fig. 7a shows typical uniformity results for several of our 3-port filter modules. Low insertion loss is maintained across all fibers in each module and repeatability of the assembly process is
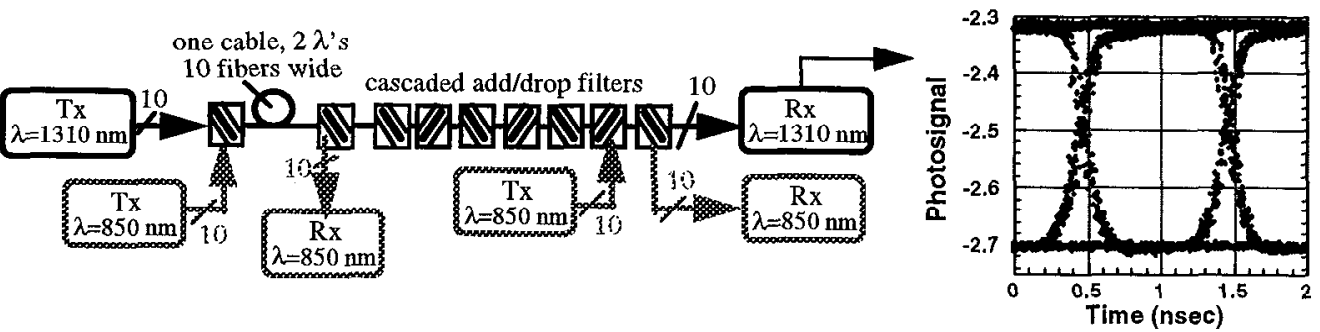

Fig. 8. Schematic of 3-port filter module cascade demonstration and eye diagram for transmission through 8 filters at $1 \mathrm{~Gb} / \mathrm{s}(\lambda=1310 \mathrm{~nm})$. Filter modules were each separated by $\sim 4 \mathrm{~m}$ fiber cable. more than one module. Fig.

$7 \mathrm{~b}$ shows uniformity results from a 2-port module, in addition to low insertion loss across all fibers, this figure shows that filter center wavelengths are maintained to $\pm 0.8 \mathrm{~nm}$.

\section{Cascaded 3-Port Filter Link Demonstration}

We demonstrated a dual wavelength link based on our 3port WDM filter modules and commercially available, bytewide transceivers (Optobahn, Optobus) [9]. $2^{23}-1$ PRBS signals at $\sim 850$ and $1310 \mathrm{~nm}$, at bit rates of 500 and 1000 $\mathrm{Mbit} / \mathrm{s} / \mathrm{fiber}$ respectively, were routed through a cascade of up to eight filters and $\sim 40 \mathrm{~m}$ of $62.5 \mu \mathrm{m}$ core GRIN fiber ribbon as shown in fig. 8. This link exhibited a total insertion loss, through all 8 filters, of $10.3 \mathrm{~dB}$ at $1310 \mathrm{~nm}$ and yielded a bit error rate below $1 \cdot 10^{-14}$, including crosstalk effects between different wavelengths and different fibers in the ribbon cables. The excess power penalty for 8 filters was only $1 \mathrm{~dB}$. Shaking of the fiber to induce potential error rate floors had no effect, indicating that the filters introduce negligible mode selective loss.

\section{Conclusions}

In conclusion, WDM filter modules for byte-wide parallel optical interconnects have been demonstrated. Filter modules suitable for add/drop multiplexing (3-port devices) and broadcast and select architectures (2-port devices) have been shown. The filter modules use low-cost plastic ferrules to provide passive alignment, and exhibit excellent transmission characteristics and channel to channel uniformity. Using multiple cavity Fabry-Perot filter designs, the 2-port devices exhibited transmission characteristics which are suitable for $10 \mathrm{~nm}$ channel spacing. Twowavelength byte-wide WDM operation was demonstrated using 3-port filter modules and commercial transceivers. Simulations indicate that our 3-port filter approach is suitable for 15-30 nm channel separations. These devices directly enable several interesting WDM interconnects such as chordal rings. Furthermore, when coupled with byte-wide multi-wavelength transceivers, this technology can be used to realize WDM interconnect fabrics with substantial sourcerouting capability and high channel bandwidth.

\section{Acknowledgments}

We thank Phil Stephan, Holly Petersen, and Bill Goward of Lawrence Livermore National Laboratory for technical assistance, and Cedric Lam and Eli Yablonovitch of UCLA for a preprint of their work. This work was performed under the auspices of the U. S. Department of Energy by Lawrence Livermore National Laboratory under contract No. W-7405Eng-48.

\section{References}

1 Wong, Y.-M., et al., 'Technology Development of a HighDensity 32-Channcl 16-Gb/s Optical data Link For optical interconnection applications for the Optoelectronic Technology Consortium (OTEC)', J. Lightwave Tcchnol. LT-13, 995-1013 (1995).

2 DeGroot, A. J., et. al. 'High Performance Parallel Processors Based on Star-Coupled WDM Optical Interconnccts', Proc. 3rd Massivcly Parallel Processing using Optical Interconnects, 1996, pp. 62-69.

3 Yanagawa, $\mathrm{H}$., et al., 'Filter-embedded Design and Its Applications to Passive Components', J. Lightwave Technol. LT-7, p. 1646-1653 (1989).

4 Winzer, G., Mahlein, H. F., and Reichelt, A.: 'Singlemode and multimode all-fiber directional couplers for WDM', Applied Optics, 1981, 20, pp. 3128-3134.

5 Deri, R. J., et al., 'Simple Fabrication of WDM Filters for Byte-Wide Multimode Cable Interconnects', Proc. 1998 Integrated Photonics Research., p. 419 (1998).

6 Satake, T. et al., 'MT Multifiber connectors and New Applications', Proc. 1994 Electronic Components \& Technology Conference., p. 994-999 (1994).

7 MacLeod, H.A., 'Thin-film Optical filters, McGraw-Hill, Colorado Springs, 1989.

8 Lam, Cedric, and Yablonovich, Eli, Private communication

9 Schwartz, D. B., et al., 'A Low-cost high-Performance Optical interconnect', IEEE Trans. Components, Packaging, and Manufacturing Technology - B, 1996, 19, pp. 532-539. 